\title{
Is Pulmonary Thromboembolism uncommon in Nigeria? A case series in a private tertiary hospital in Ogun State, Nigeria.
} Ogunkoya JO ${ }^{1}$, Oluwole $\mathrm{AO}^{2}$, Daniel $\mathrm{E}^{1}$, Ehioghae $\mathrm{O}^{1}$, Ajiboye $\mathrm{OF}^{3}$

\author{
${ }^{1}$ Respiratory Medicine Division, Department of Medicine, Babcock University Teaching Hospital, Ilishan \\ Remo, Ogun State, Nigeria. \\ ${ }^{2}$ Division of Cardiology, Department of Medicine, Babcock University Teaching Hospital, llishan-Remo, \\ Ogun State, Nigeria. \\ ${ }^{3}$ Department of Radiology, Babcock University Teaching Hospital, Ilishan-Remo, Ogun State, Nigeria.
}

Submitted: $9^{\text {th }}$ September 2020

Accepted: $27^{\text {th }}$ November 2020

Published: $30^{\text {th }}$ December 2020

\begin{abstract}
Background: Pulmonary thromboembolism is total or partial obstruction of one or more divisions of pulmonary arterial vasculature. It is a common disease presentation that is well studied and documented in the United States of America and Western Europe. It is often the result of part of a thrombotic lesion in deep veins (Deep Vein Thrombosis) elsewhere in the body most commonly the lower limbs and the pelvic region. There are few documented cases in Nigeria and sub-Saharan Africa as a result of poorly equipped hospitals and poorly trained health care personnel.

Case presentation: Twenty-Nine (29) cases were seen in a Private Tertiary institution in Ogun State, Nigeria. Computerized Tomography with Pulmonary Angiography had helped to confirm these cases, with prompt intervention thereby reducing morbidity and mortality significantly. Only 3(three) mortality was recorded out of 29 cases seen between July 2016 and June 2020.

Discussion and conclusion: Pulmonary thromboembolism is not uncommon in Nigerians and black Africans as available data previously suggested. All hands must be on deck to identify potential cases and investigate at-risk individuals who have clinical symptoms that are often misdiagnosed as other disease entities.
\end{abstract}

Key Words: Venous Thromboembolism, Pulmonary Thromboembolism, Computerized Tomography with Pulmonary Angiography, Ventilation-perfusion scanning

\section{Background}

Pulmonary thromboembolism (PTE) is a partial or complete obstruction to blood flow of a pulmonary artery or a segment of a pulmonary artery in the lungs by a clot or any substance(s) that moved from other parts of the body through the bloodstream (embolism) (1). Venous thromboembolism (VTE) is among the commonest causes of cardiovascular diseases in the world after ischemic heart disease and cerebrovascular accidents (CVA) $(1,2)$. Symptoms of a PTE may include common and has a high mortality if not treated (3).

There are unfounded beliefs that pulmonary thromboembolism is uncommon in Nigeria and black Africans. This myth is being propagated by poorly equipped health care systems, not actively on the lookout for possible cases, and most are ill-prepared to fully investigate suspected cases (4). In Africa, many patients who die from pulmonary thromboembolism were misdiagnosed premortem, a shortcoming related to the variability in clinical presentation,

Correspondence: Ogunkoya, John O.

Respiratory Medicine Division, Department of Medicine,

Babcock University Teaching Hospital, Ilishan-Remo, Ogun State, Nigeria.

+2348077503998; ogunkoyaj@babcock.edu.ng

(C) BUMJ. 2020 Open Access This article is distributed under the terms of the Creative Commons Attribution 4.0 International License 
inexperienced clinicians, inaccurate risk assessment, and lack of access to computer tomography scans (5). The United States Surgeon General estimates that PTE causes between 100,000 and 180,000 deaths in the United States alone, and singles out PTE as the most preventable cause of in-hospital death in the $U S(2,6)$. The prevalence of VTE in Africa varies between $2.4 \%$ and $9.6 \%$ in patients after surgery, and between 380 and 448 per 100000 births per year in pregnant and postpartum women (5).

Risk factors for PTE range from age, gender, geographic region, and monthly income to environmental triggers such as hypertension, diabetes, peripheral vascular disease, hyperlipidemia, chronic heart diseases, cancer, recent fracture, pregnancy, and the use of anticoagulants, hyperthyroidism (7), recent surgeries, immobilization. Others are genetic factors such as the factor $V$ Leiden mutation, low antithrombin levels, low protein $C$ levels, and low protein S levels (8). Human immunodeficiency virus infection is associated with a two to tenfold increased risk of venous thrombosis in comparison with a general population of the same age (7). Some risk factors demonstrated the strongest association with VTE such as low CD4 ${ }^{+}$ cell count especially in the presence of clinical AIDS, protein $S$ deficiency, and protein $C$ deficiency (9). PE should be included in the differential diagnosis when patients with HIVIAIDS have unexplained dyspnea or hypoxemia. In young individuals with VTE, especially men, without other identifiable risk factors for VTE, HIV should be considered as interactions between warfarin and environmental agents are possible (10). Medical Practitioners should also be alert to the potential of dangerously high or low International normalized ratios (INRs) when they are giving anticoagulants to patients with HIV infection who are undergoing antiretroviral therapy $(9,10)$. Other known risk factors for $P E$ include being overweight and obese, cigarette smoking, pregnancy, oral contraceptive pills, hormone replacement therapy, chronic illnesses like stroke, chronic heart failure, high blood pressure, and connective tissue diseases like Systemic Lupus Erythematosus, recent trauma, and burns, fractures of the hip or thigh bone and old age (11). Objective testing for PE is crucial because clinical assessment alone is unreliable and the consequences of misdiagnosis are serious (12). Failure to diagnose PE is associated with high mortality and incorrect diagnosis of the condition unnecessarily exposes patients to risks of anticoagulant therapy $(9,11)$. No single noninvasive test for $P E$ is both sensitive and specific. Some tests are good for "ruling in" PE (e.g., helical Computerized Tomography) and some tests are good for "ruling out" PE (e.g., Ddimer). Other tests can do both but often are nondiagnostic (e.g., ventilation-perfusion lung scanning). However, it is universally accepted that the gold standard for diagnosing PE is CTPA (Computerize Tomography scan with Pulmonary Angiography). It has high sensitivity and specificity PE $(1,2,4,7)$.

In a private tertiary health institution in Ogun State, Nigeria, 29 cases of Pulmonary embolism confirmed with Computer Tomography Pulmonary Angiography (CTPA) were seen between July 2018 and June 2020. This write-up discusses the presentations, investigation findings, treatments, and the outcome of some of the cases managed by the chest Unit of Babcock University Teaching Hospital, Ilishan Remo, Ogun State, Nigeria.

\section{Case presentation}

We report five patients with pulmonary thromboembolism of African descent who had initially presented with chest pain, sudden onset of breathlessness, and sudden loss of consciousness. Various evidence of pulmonary arterial obstructions ranging from filling defect in the pulmonary arteries or its branches to characteristic doughnut signs were seen in computerized tomography scans of the patients. Transthoracic echocardiographic feature of concentric hypertrophy was seen in some of the cases. Electrocardiographic findings include sinus tachycardia, right axis deviation, and characteristic large $S$ wave in lead 1, pathologic $Q$ wave in lead III, and inverted T wave in lead III (S1Q3T3). All cases responded very well to oral and parenteral anticoagulants.

\section{Case 1}

A 74-year-old healthy female presented with recurrent fainting spells of three weeks and breathlessness on exertion of two weeks' duration. She was noticed about 3 weeks earlier to be unresponsive to calls, with generalized body weakness and starring into the air. She regained full consciousness about 30 minutes later and was able to talk and walk without support. There was no history of jerking movements or loss of sphincteric control. The patient subsequently developed exertional dyspnea 2 weeks before the presentation which worsened over time to 
become present at rest about a week later. There was an associated history of palpitations and dizziness. There was a history of orthopnea and paroxysmal nocturnal dyspnea. No history of cough or pleuritic chest pain, trauma to the chest, chest tightness, or fever. There was no history of recent pelvic surgeries or long-distance travels, recurrent pregnancy loss, immobility, or history suggestive of malignancy. No abdominal or leg swelling and no history of calf pain or swelling. The patient was recently diagnosed with hypertension in a private hospital and was placed on amlodipine and hydrochlorothiazide. She was not a known Diabetic and she does not smoke cigarettes nor take alcohol. She was obese with a Body mass index (BMI) of $36.4 \mathrm{~kg} / \mathrm{m}^{2}$ but claimed to have lost about $2.5 \mathrm{~kg}$ of weight in the recent past.

On examination, she was conscious and alert, tachypneic with a respiratory rate of $46 \mathrm{cpm}$, and oxygen saturation at room air was $82 \%$. She was not pale, anicteric, not cyanosed, was mildly dehydrated with no pitting pedal edema. She desaturated to $69 \%$ after a six-minute walk test. Breathe sound was vesicular globally with coarse crepitations in the right middle lung zone. Her pulse rate was $92 \mathrm{bpm}$, regular, normal volume, synchronous with other peripheral pulses with arterial wall thickening. Blood Pressure was $120 / 70 \mathrm{mmHg}$ supine with $1^{\text {st }}$ and $2^{\text {nd }}$ heart

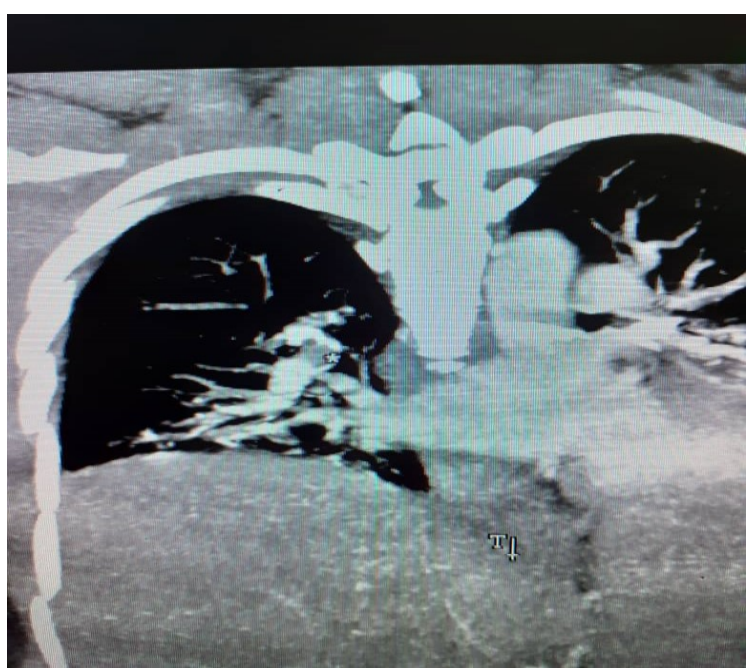

Figures 1 and 2: The Chest CTPA for the patient in Case 1

\section{Case 2}

A 40-year-old woman presented with two days' history of right-sided chest pain and a day history of difficulty in breathing. Chest pain was said to be pleuritic with a severity of 6 on a scale of 1 to 10. It was sharp and occasionally radiated to the sounds heard on auscultation of the precordium and no loud P2 and no heart murmur was heard. The Complete blood count, renal function tests, and Liver function tests were normal. The baseline $D$ dimer was greater than four, INR was 1.72. ECG showed right axis deviation, large $S$ wave in lead1, pathologic $Q$ wave in lead III, and inverted $T$ wave in lead III (SIQ3T3). Transthoracic echocardiogram showed concentric hypertrophy of the left ventricle. Chest computerized tomography with angiography showed filling defects within the right and left main pulmonary arteries and their branches nearly occluding its lumen, indicative of pulmonary embolism.

The patient was immediately placed on subcutaneous Clexane $40 \mathrm{mg}$ daily with oral warfarin for 1 week and $7.5 \mathrm{mg}$ of warfarin for 3 months with INR ranging between 2.1-2.92. She discontinued her medications after 3 months and missed 2 consecutive clinic appointments. She represented 5 months after discharge with leftsided pleuritic chest pain and fever. A chest X-ray and a new CTPA were done which were both normal. She was recommenced on oral Warfarin $5 \mathrm{mg}$ daily, Oral Amoxicillin-clavulanic acid $1 \mathrm{~g} \mathrm{bd}$ for 2 weeks, and oral clarithromycin $500 \mathrm{mg}$ bd for 10 days. The chest pain and fever resolved after a week on medications.

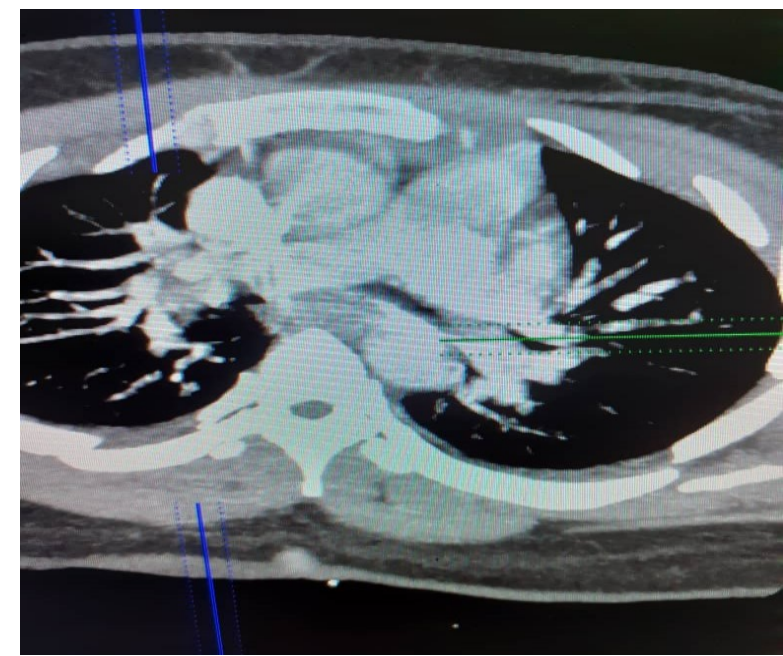

back. It was not associated with her meals. There was no history of chest trauma, no history suggestive of regurgitation of feeds, no early satiety, excessive flatulence, or excessive belching. Difficulty in breathing was first noticed a day before the presentation. It was sudden in 
onset with no known relieving or aggravating factors. There was no associated history of wheezing, cough, chest tightness. There was no history of fever, seizures, syncope, or loss of consciousness.

She delivered via a caesarian section about 9 days before the onset of chest pain on account of 2 previous caesarian sections (Para3 ${ }^{+3}$, 3Alive). She was ambulated out of bed quite early and was discharged on the fifth day after surgery. No history of recurrent pregnancy loss, no history of recent long-distance travels, and no history of previous leg pain or swelling. No dyspnea on exertion, no orthopnea or paroxysmal nocturnal dyspnea, and no palpitation. No history of facial puffiness, no reduction in urine output, no hiccups, and no pruritus. No history of use of oral contraceptive pills. She is not a known hypertensive or diabetic. She does not smoke cigarettes nor take alcohol.

On examination, she was obese (BMl= $32.7 \mathrm{~kg} / \mathrm{m}^{2}$ ), in respiratory distress with oxygen saturation at room air of $66 \%$, tachypneic (respiratory rate of 30 cycles per minute), not cyanosed, anicteric, mildly dehydrated with no pitting pedal edema. Pulse rate was 109 beats per minute, regular, normal volume, synchronous with other peripheral pulses. Blood Pressure was $114 / 89 \mathrm{mmHg}$. Jugular venous pressure was not elevated, $1^{\text {st }}$ and $2^{\text {nd }}$ heart sounds were heard, and no loud P2. There were coarse crepitations bilaterally in the lower lung zones.

Complete blood count showed normocytic hypochromic anemia with a Hemoglobin value of 9.2g. Renal and liver functions were normal. The electrocardiogram showed sinus tachycardia. Transthoracic echocardiogram was essentially normal. Chest computerized tomography with angiography showed a normal right and left pulmonary trunk with no classical filling defects within them. However, filling defects were noted at the level of the sub-segmental arteries inferiorly and bilaterally suggestive of subsegmental pulmonary embolism. Opacities were noted with air bronchogram in the posterior and basal segments bilaterally indicative of pneumonic changes.

She was placed on subcutaneous Clexane $60 \mathrm{mg}$ daily for 1 week and subsequently discharged home on oral warfarin for 3 months. She has been regular at her clinic appointment and has had no new complaints. Her last clinic visit was about 6 weeks ago. Anticoagulant was discontinued during her last clinic visit after 6 months. She expressed her desire to have another child in the future and was counseled on the need for early antenatal booking if she discovered she was pregnant. She is alive and well.
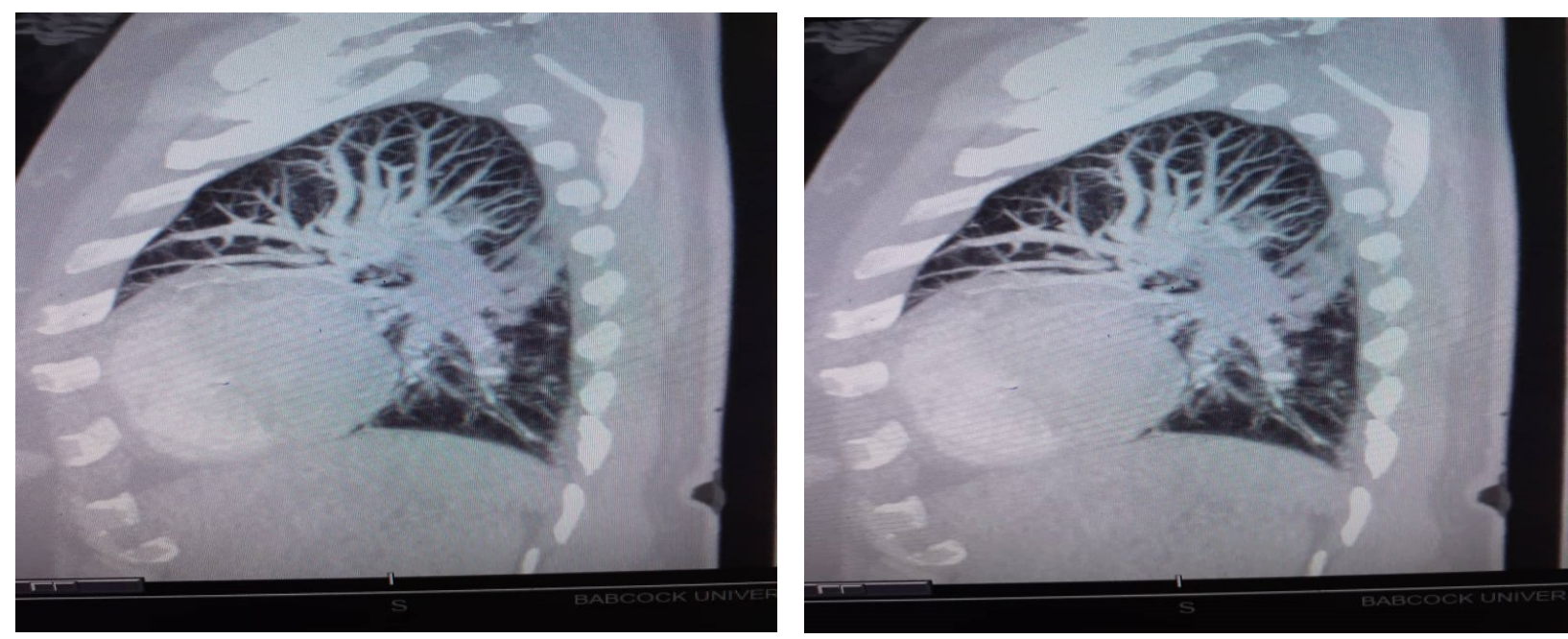

Figures 3 and 4: The Chest CTPA for the patient in Case 2

\section{Case 3}

A 32-year-old man, a healthy, non-smoking athlete presented with exertional dyspnea of a day duration and transient loss of consciousness. The exertional dyspnea started while walking over a short distance to get water to drink. He subsequently felt dizzy while driving and fainted while trying to park his car properly but eventually hitting a wall. He later had multiple episodes of dizzy spells and dyspnea which was worse on mild exertion. There was also a positive history of easy fatigability, worse on mild exertion with associated diaphoresis. No histories of chest pain, cough, calf pain, fever, abdominal or leg 
swelling. No history of long-distance travel or a sedentary lifestyle. $\mathrm{He}$ is not a known hypertensive or diabetic and not a known cancer patient. There was no history of recent surgeries or immobility and no family history of sudden cardiac death. He takes more than $30 \mathrm{~g}$ of alcohol per day. He was tachypneic with a respiratory rate of 42 cycles per minute. Oxygen saturation at room air was $82 \%$. Pulse rate was 116 beats per minute, regular, normal volume, synchronous with other peripheral pulses, no thickened arterial wall. Blood pressure was 110/92 mmHg. Jugular venous pressure was not elevated. $1^{\text {st }}$ and $2^{\text {nd }}$ heart sounds were heard with a loud P2. Chest examination was not remarkable. The abdominal examination was normal.

The Complete blood count revealed a PCV of $46 \%$. Renal function tests and Liver function tests were normal. INR was 1.4. D-dimer was negative. ECG showed sinus tachycardia, S1Q3T3, and ischemic $T$ wave changes in anteroseptal leads. Transthoracic echocardiography showed mildly dilated right atrium, right ventricles, severe tricuspid regurgitation with severe pulmonary hypertension (PASP 60.53mmgh), and concentric left ventricular hypertrophy. Right ventricular systolic function was poor with a good ejection fraction of $74.5 \%$. Chest computerized tomography with angiography showed multiple irregular intraluminal filling defects of low attenuation; some of which are surrounded by contrast are seen in the right and left pulmonary arteries and branches.

He was treated with tab Xalreto (rivaroxaban) $15 \mathrm{mg} 12 \mathrm{hrly}$ for 3 weeks, then $20 \mathrm{mg}$ daily for 2 months. He was counseled on the need to reduce his intake of alcohol. During his clinic visit 2 months after discharge, the oral anticoagulant was extended for another 4 months. There had been complete resolution of all his symptoms but the patient is struggling with alcohol addiction and he is presently abroad on treatment for alcohol addiction. He is alive and well.

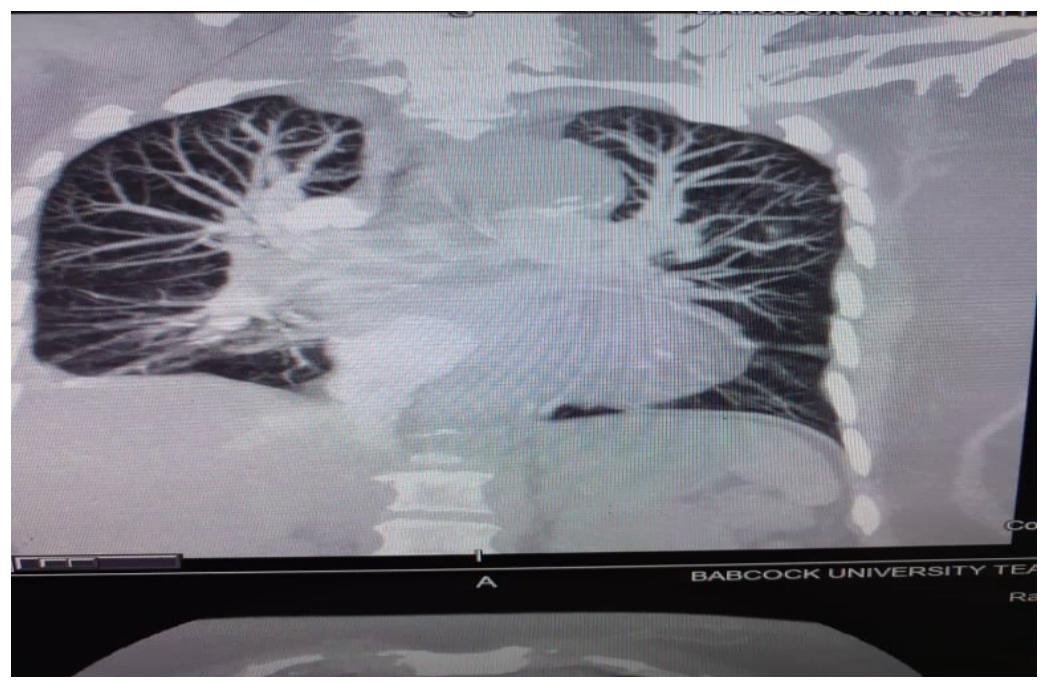

Figure 5: The Chest CTPA for the patient in Case 3

\section{Case 4}

A 58year old businessman presented with a 3week history of recurrent difficulty in breathing and cough. Sudden onset breathlessness was first noticed while working on the poultry farm. It was worse on exertion and associated with easy fatigability. Breathlessness progressively worsened with time with diaphoresis but no nausea or vomiting. He had an episode of fainting attack which was preceded by dizzy spells. No history of palpitation, orthopnea, or paroxysmal nocturnal dyspnea. No history of abdominal or leg swelling. No history of long-distance travel or sedentary lifestyle and no history of recent pelvic surgeries. He is not a known asthmatic. A nonproductive cough was noticed at about the same time as the difficulty in breathing. There was associated lateral chest pain which subsided spontaneously over a short period. No history of cancer in the patient or family members. No family history of sudden unexplained death. $\mathrm{He}$ is not a known diabetic or hypertensive. No history was suggestive of renal disease. He had 18 packyear of cigarette smoking and takes about $44 \mathrm{~g}$ of alcohol per week. He had hernia surgery one and a half months before the onset of breathlessness. On examination, he was in respiratory distress with a respiratory rate of 34 cycles per minute and 
oxygen saturation of $87 \%$ at room air, was not febrile, not cyanosed, and no bilateral pitting pedal edema. Tracheal was central, with equal chest expansion bilaterally. Breath sound was vesicular globally with expiratory rhonchi on the right middle and lower lung zones. Pulse rate: $98 \mathrm{bpm}$, regular, normal volume, synchronous with other peripheral pulses. No thickened arterial wall or locomotor brachialis. Blood pressure was $100 / 70 \mathrm{mmh}$ in the supine position. Jugular venous pressure was elevated. Precordium was normoactive with apex beat in the $6^{\text {th }}$ left intercostal space lateral to the mid-clavicular line. There was left parasternal heave. $1^{\text {st }}, 2 \mathrm{nd}$, and $3^{\text {rd }}$ heart sounds were heard at the base of the heart with a loud P2. No cardiac murmur. The abdomen was full, moves with respiration, soft and the umbilicus was flat. There was a right hypochondria tenderness. The liver was enlarged, about $6 \mathrm{~cm}$ below the right costal margin. it was firm with a smooth surface and liver span was approximately $16 \mathrm{~cm}$.

The Complete blood count, renal function tests, and Liver function tests were normal. Electrocardiogram done showed pathologic $Q$ waves in inferior as well as anteroseptal leads, with evidence of right axis deviation (RAD). Transthoracic echocardiogram showed right ventricular pressure overload, mild systolic regurgitation (peak gradient-49mmhg), IVC$2.5 \mathrm{~cm}$, estimated right atrial pressure- $15 \mathrm{mmgh}$, estimated PASP- 64mmgh, concentric left ventricular hypertrophy, good left ventricular systolic function, moderate RV dysfunction (TAPSE-10mm). Chest computed tomography pulmonary angiogram showed linear luminal filling defects $(55.2 \times 10.4 \mathrm{~mm}$ and $43 \times 9.3 \mathrm{~mm}$ in length and AP diameters) are noted straddling the pulmonary trunk, with contrast tracking in between and around them. These indicate straddle pulmonary embolism. Multiple irregular luminal filling defects of low attenuation( $\mathrm{HU}=30)$ which is outlined by contrast medium are seen within the right and left pulmonary arteries giving the railroad track sign as well as lobar and segmental arteries giving the doughnut sign all consistent with acute pulmonary embolism. There is the involvement of main pulmonary arteries, arteries to the left upper lobe, and bronchus intermedius.

The patient was treated with Subcutaneous Clexane $80 \mathrm{mg}$ daily for 1 week, oral Lasix $40 \mathrm{mg}$ daily, tab sildenafil $5 \mathrm{mg}$ TDS, and oral warfarin $5 \mathrm{mg}$ daily. He was discharged home 13 days after admission with complete resolutions of symptoms. However, the patient stopped using his medications about 3 weeks after discharge and started taking herbal drugs. He also missed his appointment scheduled for two weeks after discharge. He died at home in his sleep 5 weeks after discharge from the hospital.

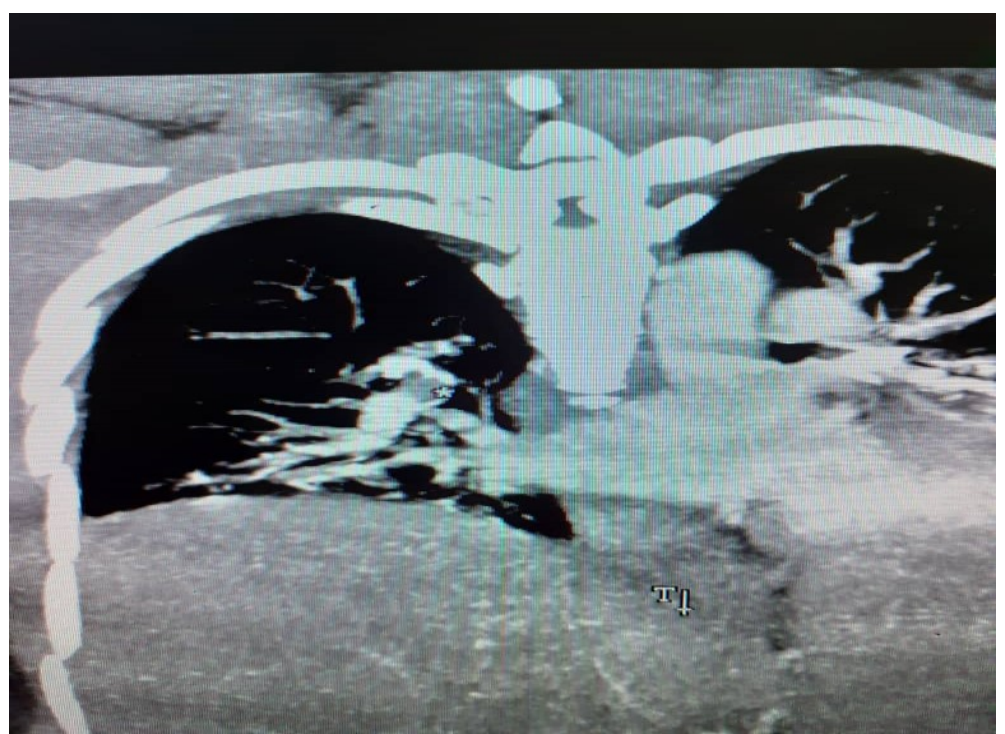

Figure 6: The Chest CTPA for the patient in Case 4

\section{Case 5}

A 56year old trader presented on account of easy fatigability of three weeks' duration, difficulty in breathing of two weeks' duration, and loss of appetite of three days' duration. She first noticed easy fatigability with a feeling of unwellness. She developed difficulty in breathing a week later which was initially on moderate exertion but 
worsened few days to the presentation. She also noticed bilateral leg swelling with associated calf pain on the right lower limb. No history of swelling of the face or abdomen. There was colicky abdominal pain and loss of appetite but no change in bowel habits. There was no history of fever or cough but the patient had a positive history of orthopnea, paroxysmal nocturnal dyspnea, and palpitations. She is not a known hypertensive or diabetic patient. She was recently diagnosed with heart failure at the referral center and has no family history of heart disease. No history of recent long-distance travel. She takes locally brewed alcohol about $15 \mathrm{~g}$ per day. No history of tobacco use.

Examination showed a middle-aged woman in obvious respiratory distress with oxygen saturation of $56 \%$ in room air which improved to $95 \%$ on 13 Liters per minute of oxygen via facemask. She was anicteric, not pale, not dehydrated. There were multiple hyperpigmented patches on the face and skin and pitting pedal edema up to the ankle bilaterally. There was swelling and tenderness of the calf region on the right. Pulse rate was 117 beats per minute, small volume, regular and synchronous with other peripheral pulses. Blood pressure was 109/85 $\mathrm{mmHg}$ and the jugular venous pressure was raised above the angle of the jaw. The apex beat was in the Left $6^{\text {th }}$ intercostal space, anterior axillary line. S1, S2, S3 were present with a pan systolic murmur loudest at the apex. Respiratory rate was 34 cycles per min and vesicular breath sounds were heard globally with coarse crepitations in the right lung base. The abdomen was full with mild epigastric tenderness and there was no organomegaly.

Complete blood count, Electrolytes, Urea \&Creatinine, lipid profile, and abdominal ultrasound were all normal. Chest radiograph showed cardiomegaly with evidence of pulmonary congestion. Electrocardiography revealed sinus tachycardia with left atrial enlargement and left axis deviation. Echocardiography showed mild left ventricular hypertrophy, dilated right ventricle, global LV wall hypokinesia, moderate tricuspid regurgitation with severe pulmonary hypertension. Grade I Left ventricular diastolic dysfunction. Good right ventricular systolic function (TAPSE $-2.3 \mathrm{~cm}$ ). Borderline left ventricular systolic function (EF $50 \%$ ). Doppler ultrasonography confirmed the presence of thrombi with partial occlusion in the upper half of the right popliteal vein. Computed Tomography Pulmonary Angiography revealed a filling defect of about 5.07 noted arising from the origin of the left upper lobe segmental branches from the left main pulmonary trunk. Other filling defects were seen in the proximal portion of the left lower lobe segmental branches and the right main pulmonary artery measuring $3.5 \mathrm{~cm}$ in length at the origins of the segmental branches of the right middle and right lower lobes.

She was treated with amlodipine, indapamide, rivaroxaban, and telmisartan tablets. The patient was out of failure within 2 weeks after admission and was discharged home on tab rivaroxaban $20 \mathrm{mg}$ daily, tab amlodipine $5 \mathrm{mg}$ daily, tab indapamide $1.5 \mathrm{mg}$ daily, and telmisartan $80 \mathrm{mg}$ daily. She has been regular at clinic appointments. There are no new complaints. Repeat echocardiography revealed she still has mild left ventricular hypertrophy, dilated right ventricle, and moderate tricuspid regurgitation. There is also moderate pulmonary hypertension with good right ventricular systolic function (TAPSE $-2.2 \mathrm{~cm}$ ) and EF - 54\%). She is alive and well on all prescribed oral medications.
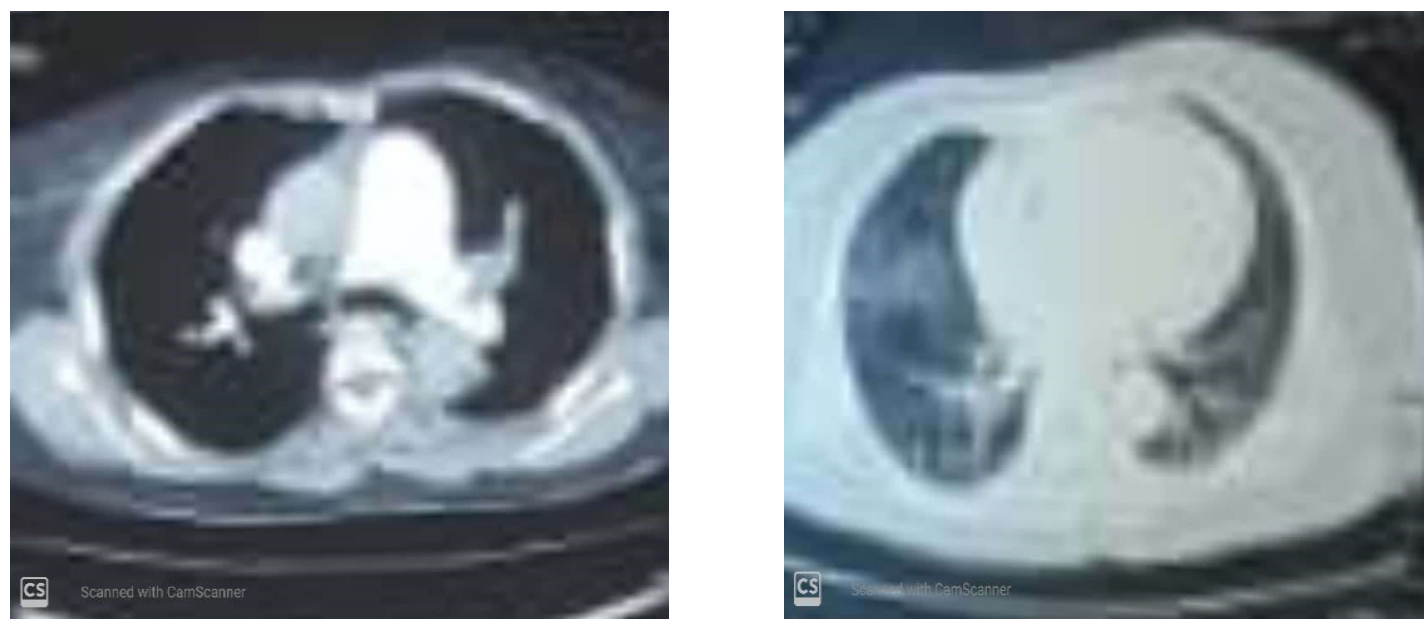

Figures 7 and 8: The Chest CTPA for the patient in Case 5 


\section{Discussion}

This case series described five cases of pulmonary embolism with different modes of presentation. The cases were managed using current clinical practice guidelines resulting in early diagnosis and exclusion of differentials. A high index of suspicion associated with the immediate availability of CTPA and other diagnostic tests tools such as d-dimer, CTPA, Compressive ultrasonography, Electrocardiography, and Echocardiography resulted in a high survival rate seen in our hospital.

This case series showed the clinical profile of patients with pulmonary embolism who were admitted, investigated, and treated at our hospital. The availability of CTPA and other diagnostic tests allowed us to confirm PE cases and exclude other differential diagnoses. The prevalence of PE in Sub Saharan Africa is not well known but PE is not as rare in Sub Saharan Africa as previously thought (13). In our center, 1 out of every 926 patients seen in the Emergency room was diagnosed and treated for PE. The prevalence of VTE/PE in Africa according to Danwang $C$ et al (5) is high following surgery, pregnancy, and postpartum. However, only one case in this series was associated with pregnancy.

The most common symptoms in our series were breathlessness (all cases), fainting spells (cases $1,3 \& 4)$, chest pain, and cough. This is similar to findings in the Angolan study (4) and the JASPER study (14). The symptoms of pulmonary embolism are in most cases non-specific, to ensure prompt diagnosis and treatment, a high index of suspicion is needed especially in patients with a high risk of cardiovascular diseases. A sudden occurrence of breathlessness may suggest PE (15).

Cigarette smoking, obesity, increased BMI, hypertension, chronic heart diseases, and pregnancy/ postpartum has been associated with an increased incidence of PE (11). In this case series, cases 1 and 2 had obesity and this may have contributed to the development of PE in them. Case 4 had a significant history of cigarette smoking and heart failure while case 2 was in the postpartum period. These could have contributed in variable degrees to the development of PE in these patients. This is similar to findings by Danwang $C$ et $\mathrm{al}^{5}$ and other studies $(13,16,17)$. One patient (case 3 ) had no identifiable risk factor(s) but there was a history of significant intake of alcohol which is a known cardiovascular risk factor. This suggests that there are unknown risk factors associated with the susceptibility of individuals to PE (18).

In our case series, the most important diagnostic tools for PE were Computerized tomography pulmonary angiography (CTPA), electrocardiogram (ECG), and echocardiogram $(E C H O)$. This case series underscores the importance of CTPA in the diagnosis of PE. A study by Tambe $\mathrm{J}$ et al. (13) underlines the importance of CTPA in prompt diagnosis of PE. This further affirms the already fact that CTPA is the gold standard for the diagnosis of PE. The most common ECG finding was sinus tachycardia (cases 2, 3 \&5) followed by the characteristic feature of large $S$ wave in lead1, pathologic $Q$ wave in lead III, and inverted T wave in lead III (SIQ3T3) (cases 1\&3). This is similar to the finding in the Angola study (4). The most common abnormality on the echocardiogram was left ventricular hypertrophy (cases 1, 3 \&4). This finding shares no similarity with other studies in which right atrial enlargement and right axis deviations were the two most common ECG findings $(3,4,7,13)$. However, it is an important tool in the investigation and management of $\mathrm{PE}^{4}$. It is non-invasive and inexpensive.

The majority of the cases were treated with low molecular weight heparin and warfarin. They prevent the extension of old thrombi and the formation of new ones. None of the patients in this case series received thrombolytic therapy. This is similar to the result found in the EMEP (Estudo Multicêntrico de Embolia Pulmonar) study (19), in which thrombolytic therapy was used in only $15 \%$ of patients. Multiple studies showed that there is no significant difference in the length of stay on admission in the hospital, morbidity, and mortality between patients managed with anticoagulants and thrombolytic (17).

From the hospital record, out of the 29 confirmed PE patients seen over four years in our center, only 2 died on admission. The third patient died at home after discharge, probably as a result of non-compliance with medications. In-hospital death seen at our center is lower than the death rate reported in most studies (19). This may be because the patient presented early, promptly diagnosed with the aid of CTPA, and treatment instituted immediately. In line with this report, the patient that died in this study presented late (3 weeks after onset of symptoms) and was not compliant with medication after discharge.

The use of CTPA for prompt diagnosis PE in all patients in this study may be one of the reasons why in-hospital death was low as against reports 
from other studies in Africa $(4,10,13)$. Institution availability and easy access to CTPA may lead to prompt detection of the disease in various parts of Africa thereby leading to a significant decrease in rising cardiovascular mortality which is on the increase in Africa (6).

\section{Conclusion}

Pulmonary thromboembolism is not uncommon in Nigerians and black Africans as available data previously suggested. All hands must be on deck to identify potential cases and investigate at-risk individuals who have clinical symptoms that are often misdiagnosed as other disease entities. The role of CTPA in the Diagnosis of PE cannot be overemphasized. Efforts must therefore be put in place to make it widely available and easily accessible to Africans and particularly Nigerians.

\section{List of Abbreviations}

VTE: Venous Thromboembolism

PE/ PTE: Pulmonary Embolism/Pulmonary Thromboembolism

CHD: Chronic Heart Diseases

CTPA: Computerized Tomography Pulmonary Angiography

\section{Declarations}

Ethics approval and consent to participate Written and verbal informed consent to publish without the patients' names attached was obtained from all participants.

\section{Consent for publication}

The authors hereby give consent for the publication of our work under the creative commons CC Attribution-Noncommercial 4.0 license.

\section{Availability of data and materials}

The data and materials associated with this research will be made available by the corresponding author upon reasonable request.

\section{Competing interests}

The authors have declared no conflict of interest.

\section{Funding}

The authors received no research funding.

\section{Authors' contributions}

OJO was responsible for the concept, literature search, data collection, drafting, and review of the manuscript. OAO contributed to the conceptualization, literature search, drafting, and review of the paper. Also, DE participated in the drafting and critical review of the manuscript. AOF participated in the drafting and critical review of the text. All authors approve the final draft and declare that it represents honest work.

\section{Acknowledgment}

We express a special thanks to the head of the department of medical records at Babcock University Teaching Hospital, Mr. Adedayo, for the assistance rendered during this work in ensuring unfettered access to the medical records of the patients included in this study.

\section{References}

1. Ngome O, Rohacek M. Point-of-Care Ultrasound: A Useful Diagnostic Tool in Africa. Praxis. $2020 \quad$ Jun 9. https://doi.org/10.1024/1661-8157/a003495

2. Samuel Z.G. Deep Venous Thrombosis and Pulmonary thromboembolism. Harrison's Principles of internal medicine. In: Fauci AS, Kasper DL, Hauser SL, Longo DL, Jameson JL, eds. $18^{\text {th }}$ editions 2008; 2: 2170-2177.

3. Creager M, Loscalzo J, Beckman JA. Vascular Medicine E-Book: A Companion to Braunwald's Heart Disease. Elsevier Health Sciences; 2012 Aug 30.

4. Manuel A, Peralta T, Abel Salas JR, Sabola P, Baião $D$, Martins $T$, Júnior $A P$, Aufico $A$, Africano R, Silva A, Sotolongo C. Clinical profile, management and outcomes of patients with pulmonary embolism: a retrospective tertiary centre study in Angola. Cardiovascular journal of Africa. 2017 Nov;28(6):356. https://doi.org/10.5830/cvja2017-017

5. Danwang C, Temgoua MN, Agbor VN, Tankeu AT, Noubiap JJ. Epidemiology of venous thromboembolism in Africa: a systematic review. Journal of Thrombosis and Haemostasis. 2017 Sep;15(9):1770-81. https://doi.org/10.1111/jth.13769

6. Raskob GE, Angchaisuksiri P, Blanco AN, Buller H, Gallus A, Hunt BJ, Hylek EM, Kakkar A, Konstantinides SV, McCumber M, Ozaki Y. Thrombosis: a major contributor to global disease burden. Arteriosclerosis, thrombosis, and vascular biology. $2014 \mathrm{Nov}$;34(11):236371. https://doi.org/10.1111/jth.12698

7. Lin HC, Yang LY, Kang JH. Increased risk of pulmonary embolism among patients with hyperthyroidism: a 5-year follow-up study. Journal of Thrombosis and Haemostasis. 2010 
https://doi.org/10.1111/j.1538-

7836.2010.03993.x

8. Bezemer ID, van der Meer FJ, Eikenboom JC, Rosendaal FR, Doggen CJ. The value of family history as a risk indicator for venous thrombosis. Archives of internal medicine. 2009 Mar 23;169(6):610-5. https://doi.org/10.1001/archinternmed.2008.5 89

9. Bibas M, Biava G, Antinori A. HIV-associated venous thromboembolism. Mediterranean journal of hematology and infectious diseases. 2011;3(1). https://doi.org/10.4084/mjhid.2011.030

10. Ndadza A, Thomford NE, Mukanganyama S, Wonkam A, Ntsekhe M, Dandara C. The genetics of warfarin dose-response variability in Africans: an expert perspective on past, present, and future. Omics: a journal of integrative biology. 2019 Mar 1;23(3):152-66. https://doi.org/10.1089/omi.2019.0018

11. Kearon C. Natural history of venous thromboembolism. InSeminars in vascular Medicine 2001 (Vol. 1, No. 01, pp. 027-038). Copyright $\odot 2001$ by Thieme Medical Publishers, Inc., 333 Seventh Avenue, New York, NY 10001, USA. Tel.:+ 1 (212) 5844662. https://doi.org/10.1055/s-2001-14539

12. Pineda L A, Hathwar V S, Grant B J. Clinical Suspicion of Fatal Pulmonary Embolism. Chest 2001; 120:791-5. Pineda LA, Hathwar VS, Grant BJ. Clinical suspicion of fatal pulmonary embolism. Chest. $2001 \mathrm{Sep}$ 1;120(3):791-5. https://doi.org/10.1378/chest.120.3.791

13. Tambe J, Moifo B, Fongang E, Guegang E, Juimo AG. Acute pulmonary embolism in the era of multi-detector CT: a reality in subSaharan Africa. BMC medical imaging. 2012 Dec;12(1):1-6. https://doi.org/10.1186/14712342-12-31

14. Nakamura M, Fujioka $\mathrm{H}$, Yamada N, Nakano $T$, Sakuma $M$, Okada $O$, Kuriyama $T$, Nakanishi N, Miyahara Y, Kunieda T, Sugimoto T. Clinical characteristics of acute pulmonary thromboembolism in Japan: results of a multicenter registry in the Japanese Society of Pulmonary Embolism Research. Clinical cardiology. 2001 Feb;24(2):132-8. https://doi.org/10.1002/clc.4960240207

15. Centers for Disease Control and Prevention. International classification of diseases, ninth revision, clinical modification (ICD-9-CM).

16. Goldhaber SZ, Visani L, De Rosa M. Acute pulmonary embolism: clinical outcomes in the
International Cooperative Pulmonary Embolism Registry (ICOPER). The Lancet. 1999 Apr 24;353(9162):1386-9. https://doi.org/10.1016/s01406736(98)07534-5

17. Volschan A, Albuquerque DC, Tura BR, Knibel FM, Souza PC, Toscano ML. Pulmonary embolism: multicenter registry in tertiary hospitals. Revista Brasileira de terapia intensiva. $2009 \quad$ Aug;21(3):237-46. https://doi.org/10.1590/s0103507x2009000300002

18. Goldhaber SZ. In: Kasper D, Hauser S, Jameson JL Deep venous thrombosis and pulmonary thromboembolism, Fauci A, Longo DL, Loscalzo JL (eds). Harrison's Principles of Internal Medicine. 19th eds. London: McGraw-Hill, 2015: 1631-1637. https://doi.org/10.1111/j.14455994.2008.01837.x

19. Volschan A, Albuquerque DC, Tura BR, Knibel FM, Souza PC, Toscano ML. Pulmonary embolism: multicenter registry in tertiary hospitals. Revista Brasileira de terapia intensiva. $2009 \quad$ Aug;21(3):237-46. https://doi.org/10.1590/s0103$507 \times 2009000300002$ 